\title{
Effects of circadian rhythm disorder on body composition in women aged $31-40$ years
}

\author{
Ran Meng ${ }^{1 \#}$, Yidan Cao ${ }^{2 \#}$, Yong Kong ${ }^{3 \#}$, Kai Wang ${ }^{2}$, Zengkun Yang ${ }^{4}$, Yingying Jia ${ }^{2}$, Chen Dong ${ }^{1}$, \\ Haiyun Duan ${ }^{2}$, Mengshu Han $^{2}$
}

${ }^{1}$ School of Sports and Social Sciences, Shandong Sport University, Jinan, China; ${ }^{2}$ College of Physical Education of Jiangsu Normal University, Xuzhou, China; ${ }^{3}$ Department of Physical Education, China University of Petroleum, Qingdao, China; ${ }^{4}$ Secondary School Affiliated to Shandong Normal University, Jinan, China

Contributions: (I) Conception and design: R Meng, Y Cao, Y Kong, K Wang; (II) Administrative support: Y Cao, K Wang, Yi Jia; (III) Provision of study materials or patients: R Meng, Y Cao, Y Kong, Z Yang, C Dong; (IV) Collection and assembly of data: All authors; (V) Data analysis and interpretation: Z Yang, Y Jia, C Dong, K Wang, H Duan, M Han; (VI) Manuscript writing: All authors; (VII) Final approval of manuscript: All authors.

"These authors contributed equally to this work as co-first authors.

Correspondence to: Kai Wang. College of Physical Education of Jiangsu Normal University, 101 Shanghai Road, Xuzhou, China.

Email: wangkai-liaoda@163.com.

Backgrounda Circadian rhythm disorder caused by lack of sleep, day-night reversal, or staying up late, can cause irreparable damage to the body, a significant increase in both the systolic and diastolic blood pressure of women in this age bracket. The purpose of this study was to investigate the effects of circadian disruption on body composition and insulin resistance in women aged 31-40 years.

Methods: Thirty female with circadian rhythm disorder and employed of the Foot Bath Club in Qufu city were selected as the research subjects, and a body composition analyzer was used to test the subjects' body composition using bioelectrical impedance analysis. Serum melatonin (MT) levels were determined by enzyme-linked immunosorbent assay (ELISA), and blood glucose and insulin levels were detected by glucose oxidase and chemiluminescence assays, respectively.

Results: The results showed that circadian rhythm disorder caused a rise in blood pressure, whereas body water, especially lower limb water and intra- and extracellular fluid, decreased significantly. Circadian disruption also resulted in decreased muscle mass, protein quality, and melatonin production, but increased visceral fat levels and insulin resistance.

Conclusions: This study has demonstrated that circadian rhythm disorders are responsible for changes in body composition, melatonin levels, and insulin resistance in women aged 31-40 years and may be a risk factor for cardiovascular disease and diabetes.

Keywords: 31-40 years; women; circadian rhythm disorders; body composition; insulin resistance

Submitted Oct 19, 2020. Accepted for publication Jan 11, 2021.

doi: 10.21037/apm-20-2267

View this article at: http://dx.doi.org/10.21037/apm-20-2267

\section{Introduction}

Across a person's lifetime, sleep is essential for optimal functioning of every bodily organ, because sleep itself is a process of rest and recovery and plays a vital role in regulating the balance of physical and mental health.
However, circadian rhythm disorder or imbalance caused by lack of sleep, day-night reversal, or staying up late, can cause irreparable damage to the body (1). For instance, sleep deprivation can cause a number of serious health problems including obesity, type 2 diabetes, hypertension, and cardiovascular disease $(2,3)$. In addition, some sleep- 
deprivation studies of both humans and mice have shown increased food intake $(4,5)$, whereas sleep-deprived rats exhibit strong catabolism and energy expenditure causing significant weight loss $(6,7)$. More importantly, people experiencing inadequate sleep demonstrate an increase in body mass index (BMI) and a decrease in self-evaluated health $(8,9)$. Studies have found that circadian rhythm disorder can cause increased mean arterial pressure and decreased sleep efficiency of subjects significantly (10). Some studies have also found that the disturbance of circadian rhythm changes the 24-hour blood pressure of the subjects (11). Moreover, studies have also shown that circadian rhythm disruption can increase blood pressure by causing a decrease melatonin level in the body $(12,13)$. At present, many people are engaged in jobs that require night work, such as hospital and factory shift workers and various nightlife workers, which result in lack of sleep or circadian rhythm disorders. In this study, we recruited women aged 31-40 years to study the effects of circadian rhythm disorder on their body composition and insulin resistance, in order to highlight the harmfulness of circadian rhythm disorder to women's health. We present the following article in accordance with the MDAR reporting checklist (available at http://dx.doi.org/10.21037/apm-20-2267).

\section{Methods}

\section{Research objects and methods}

Thirty female staff members of the Foot Bath Club in Qufu city who were aged 31-40 years were selected for the current study, whose daily working hours are generally from 20:00 to 4:00 in the morning of the next day. After screening for inclusion and exclusion criteria, a final sample of 25 healthy adult women were included as the circadian rhythm disorder group. A further 20 women in the same age range without circadian rhythm disorder were selected as the normal rhythm group. None of the study participants had a long-term exercise or fitness history. All participants were given comprehensive information about the safety of the testing and the lack of risk to their physical health. All participants were required to complete a sleep status questionnaire and provided informed consent. The experiment was approved by the school ethics committee of Jiangsu Normal University. All procedures performed in this study involving human participants were in accordance with the Declaration of Helsinki (as revised in 2013).

\section{Screening criteria}

Inclusion criteria were as follows: (I) aged 31-40 years old; (II) female adults in good health; (III) no evidence of disease or other conditions that would adversely affect the test process and results; (IV) agreeable to volunteer for the study.

Exclusion criteria were as follows: (I) any physical disability or limb deformities; (II) any severe cardiovascular, endocrine, or metabolic diseases; (III) use of any menstrual abnormality medications; (IV) individuals unable to walk; (V) bone fractures; (VI) individuals unwilling to complete the research requirements; (VII) a long-term exercise history or employment as a sports trainer; (VIII) any neuromuscular disease that affects neuromuscular function.

\section{Index detection}

The body composition analyzer BD.ZEUS 9.9 (JAWON Medical Co. Ltd., Korea) was used to test the body composition of the subjects via Bioelectrical Impedance Analysis (BIA). Prior to testing, subjects were asked to empty their bowel and bladder, wear light clothing, remove any accessory jewelry (especially close-fitting metal jewelry), and remain in a physically restful state. The instrument's platform and handle were disinfected before use. After the subject's hands and feet were wiped with physiological electrolysis paper, the subject stood barefoot on the electrode contact surface of the instrument platform. After completion of the body mass measurement, the personal information of the subject was accurately input according to the instrument instructions. The subject held the electrode handle with both hands and activated the electrode switch button themselves, placing their arms on both sides of the body at an angle of $15^{\circ}$ to the torso. In this procedure, the harmless microcurrent of the human body is used to measure the impedance of the current flowing through various parts of the body. After approximately 1 minute, the instrument completed the test and automatically analyzed and output the test results, which included various indicators of body composition.

Measurement indicators included body weight, lean body weight, total body water content, trunk and limb water content, intracellular fluid, extracellular fluid, total body muscle mass, trunk and limb muscle mass, body fat mass, visceral fat mass, visceral fat area, subcutaneous fat mass, waist-to-hip ratio, visceral fat level, body fat percentage, 
Table 1 Comparison of body weight and blood pressure between the circadian rhythm disorder group and the normal rhythm group

\begin{tabular}{lcccc}
\hline \multirow{2}{*}{ Group } & Case & Body weight & \multicolumn{2}{c}{ Blood pressure $(\mathrm{mmHg})$} \\
\cline { 4 - 5 } & & & SBP & DBP \\
Normal rhythm group & 20 & $59.28 \pm 9.55$ & $104 \pm 13$ & $65 \pm 7$ \\
Circadian rhythm disorder group & 25 & $57.05 \pm 4.67$ & $121 \pm 10^{*}$ & $72 \pm 9^{*}$ \\
\hline
\end{tabular}

${ }^{*}, \mathrm{P}<0.05$. SBP, systolic blood pressure; DBP, diastolic blood pressure.

basal metabolic rate, protein quality, mineral quality, and a further 20 body composition indicators.

Both the circadian rhythm disorder group and the normal rhythm group were required to fast for more than 10 hours, and then $5 \mathrm{~mL}$ of venous blood was drawn between 07:30-09:30 a.m., and placed into a dry test tube with $40 \mu \mathrm{g}$ edetate disodium (10\% EDTA-2Na) and mixed immediately for anticoagulation. After standing at room temperature for $30 \mathrm{~min}$, the blood samples were centrifuged at 3,000 r/min for $10 \mathrm{~min}$. Finally, approximately $2 \mathrm{~mL}$ of serum was separated and stored in a refrigerator at $-80{ }^{\circ} \mathrm{C}$ for further testing. The patient's serum melatonin (MT) level was tested by enzyme-linked immunosorbent assay (ELISA). Blood glucose and insulin levels were tested using glucose oxidase and chemiluminescence assay methods, respectively, and then insulin resistance (HOMA-IR) was estimated based on the results of the fasting blood glucose and insulin levels. All reagents were purchased from Wuhan Boster Biological Co., Ltd (Wuhan, China).

\section{Statistical analysis}

All valid data and results were classified and sorted. The index data obtained from the body composition testing were exported to the body composition analysis software. All valid data were classified and counted in Microsoft Excel, and are expressed as means \pm standard deviation $(\bar{x} \pm \mathrm{s})$. Statistical analysis was conducted with SPSS 20.0 software (IBM, USA). Independent between-group samples were compared by $t$-test, and results with $\mathrm{P}<0.05$ were considered statistically significant.

\section{Results}

Comparison of body weight and blood pressure between the circadian rhythm disorder group and the normal rhythm group

As shown in Table 1, the body weight of the circadian rhythm disorder group was somewhat lower than that of the normal rhythm group, but the difference was not statistically significant. However, the blood pressure of the circadian rhythm disorder group was significantly increased compared with the normal rhythm group $(\mathrm{P}<0.05)$. In the rhythm disorder group, the increase of systolic blood pressure (SBP) was greater than that of diastolic blood pressure (DBP).

\section{Comparison of the body fluid indices between the circadian rhythm disorder group and the normal rhythm group}

As shown in Figure 1, the total body water and total trunk water of females in the circadian rhythm disorder group showed a downward trend compared with the normal rhythm group, and the difference in total body water was statistically significant $(\mathrm{P}<0.05)$. In the comparison of upper and lower limb water, the circadian rhythm disorder group showed a decrease. There was no significant difference in the changes of the left and right upper limbs, but the reduction in the left and right lower limbs was significantly different $(\mathrm{P}<0.05)$. In the circadian rhythm disorder group, the intra- and extracellular fluid were significantly lower than that in the normal rhythm group $(\mathrm{P}<0.05)$. The above results indicated that circadian rhythm disorders can cause significant changes in total body water, and intra- and extracellular fluid in women aged $31-40$ years.

\section{Comparison of muscle mass in various parts of the body between the circadian rhythm disorder group and the normal rhythm group}

As shown in Figure 2, compared with the normal rhythm group, the total body muscle mass and total trunk muscle mass of the circadian rhythm disorder group showed some decrease, but there was no significant difference between the groups in total trunk muscle mass. However, the total muscle mass of the body was significantly different between the two groups $(\mathrm{P}<0.05)$. The comparison of the muscle mass of the upper and lower limbs revealed a lower mass in 


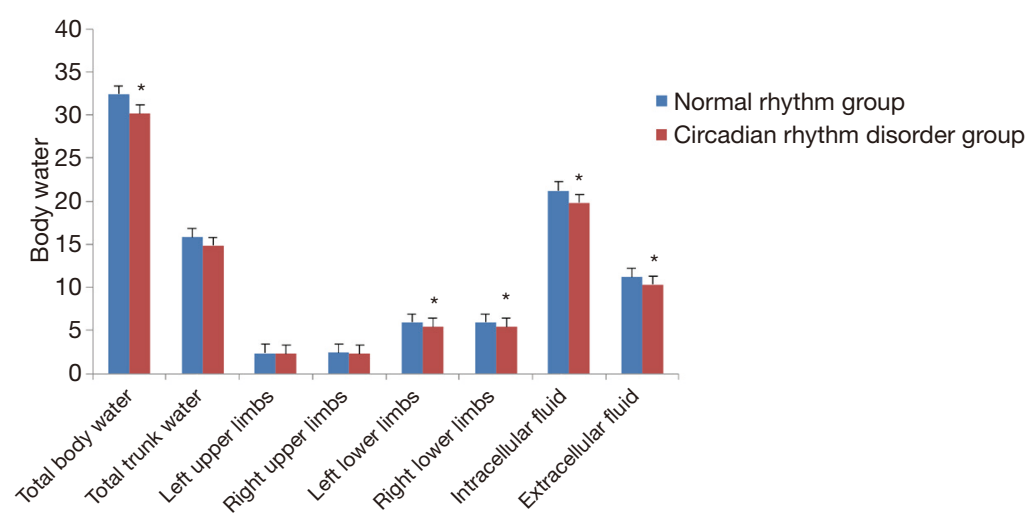

Figure 1 Comparison of body water between the circadian rhythm disorder group and the normal rhythm group $(\bar{x} \pm \mathrm{s}) .{ }^{*}, \mathrm{P}<0.05$ indicates a significant difference compared with the normal rhythm group.

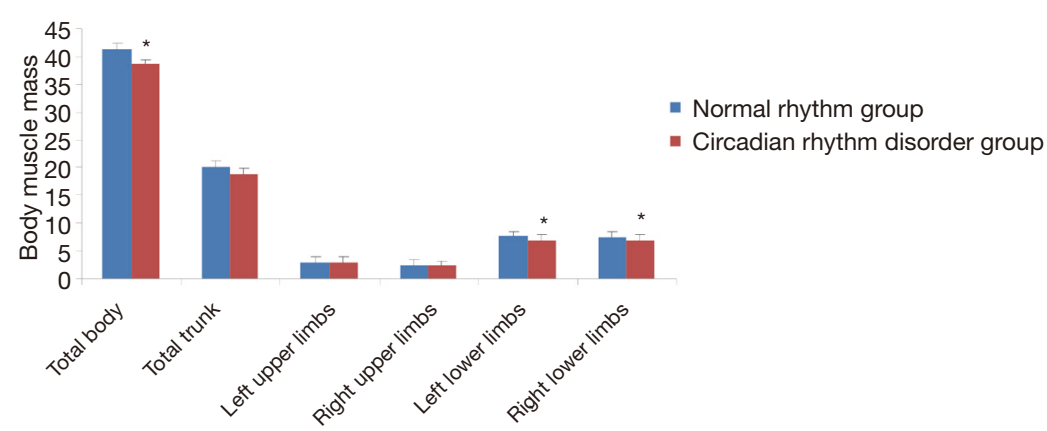

Figure 2 Comparison of body muscle mass between the circadian rhythm disorder group and the normal rhythm group. *, $\mathrm{P}<0.05$ indicates a significant difference compared with the normal rhythm group.

the circadian rhythm disorder group than that in the normal rhythm group. However, there was no significant difference in the muscle mass of the left and right upper limbs, while the reduction in the muscle mass of the left and right lower limbs was significantly different between the two groups $(\mathrm{P}<0.05)$, indicating that circadian rhythm disorder causes changes in the muscle mass of women aged 31-40 years, and the changes in total body muscle mass and lower limb muscle mass are particularly significant.

\section{Comparison of body fat mass between the circadian rhythm disorder group and the normal rhythm group}

As shown in Figure 3, compared with the normal rhythm group, the body fat mass of the circadian rhythm disorder group was significantly higher $(\mathrm{P}<0.05)$, and the amount of visceral fat was also significantly increased $(\mathrm{P}<0.05)$. The level of visceral fat and the percentage of body fat were both higher in the circadian rhythm disorder group than in the normal rhythm group, but did not reach statistical significance. However, the amount of subcutaneous fat in the circadian rhythm disorder group was significantly lower compared with the normal rhythm group $(\mathrm{P}<0.05)$, which indicates that circadian rhythm disorder can cause changes in the body fat of women aged 31-40 years, especially in visceral fat.

\section{Comparison of body basal metabolic rate, protein quality, and mineral quality indicators between the circadian rhythm disorder group and the normal rhythm group}

As shown in Figure 4, compared with the normal rhythm group, the basal metabolic rate, protein quality and mineral quality of the circadian rhythm disorder group all showed a reduced trend, but only the protein quality showed a statistically significant difference $(\mathrm{P}<0.05)$. The results indicate that circadian rhythm disorder can lead to the decline of the basal metabolic rate, protein quality and mineral quality in women aged 31-40 years, and especially in protein quality. 


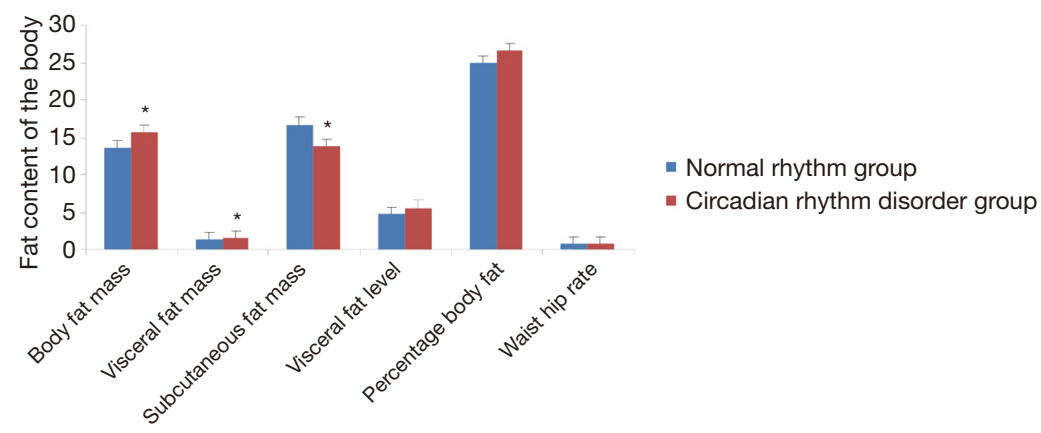

Figure 3 Comparison of fat content in various parts of the body between the circadian rhythm disorder group and the normal rhythm group. *, $\mathrm{P}<0.05$ indicates a significant difference compared with the normal rhythm group.

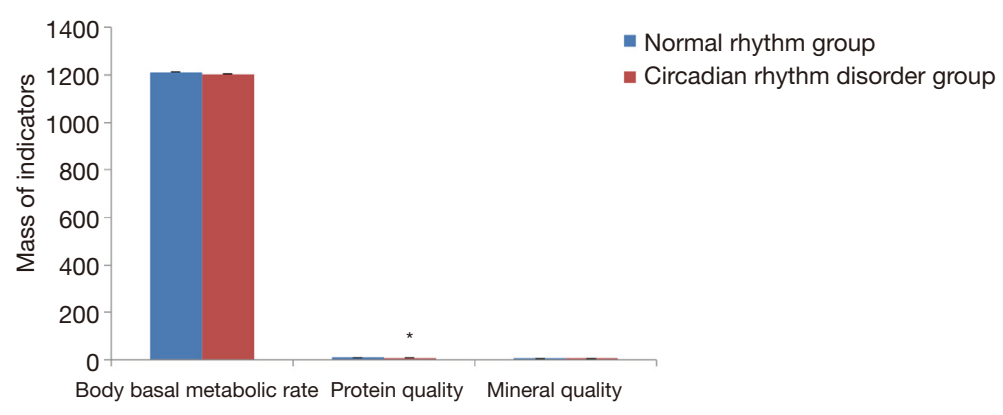

Figure 4 Comparison of the basal metabolic rate, protein mass and mineral mass between the circadian rhythm disorder group and the normal rhythm group. * $\mathrm{P}<0.05$ indicates a significant difference compared with the normal rhythm group.

Table 2 Comparison of melatonin and insulin resistance (HOMA-IR) between the circadian rhythm disorder group and the normal rhythm group

\begin{tabular}{lccc}
\hline Group & Case & Melatonin/(pg· $\left.L^{-1}\right)$ & Insulin resistance \\
\hline Normal rhythm group & 20 & $66.5 \pm 8.1$ & $1.17 \pm 0.26$ \\
Circadian rhythm disorder group & 25 & $53.1 \pm 6.3^{*}$ & $1.79 \pm 0.69^{\star}$ \\
\hline
\end{tabular}

*, $\mathrm{P}<0.05$.

\section{Comparison of melatonin and insulin resistance (HOMA- $I R)$ between the circadian rhythm disorder group and the normal rhythm group}

As shown in Table 2, compared with the normal rhythm group, the melatonin level of the circadian rhythm disorder group was significantly lower $(\mathrm{P}<0.05)$, and the insulin resistance was significantly higher $(\mathrm{P}<0.05)$, indicating that circadian rhythm disorder can cause significant changes in melatonin levels and insulin resistance in women aged 31-40 years old.

\section{Discussion}

This study finds that circadian rhythm disorder does not have a significant impact on the weight of women aged 31-40 years, who only displayed marginal weight loss. However, circadian rhythm disorder causes a significant increase in both the systolic and diastolic blood pressure of women in this age bracket. Preliminary experimental research from Scheer et al. (10) with non-shift workers found that circadian rhythm disorder caused a significant increase in the average arterial pressure of the subjects 
$(+3 \%, \mathrm{P}=0.001)$, and also caused a significant decrease in their sleep efficiency $(-20 \%, \mathrm{P}<0.002)$. Morris et al. (11) also studied non-shift workers and observed a similar phenomenon of changes in 24-hour blood pressure following circadian rhythm misalignment. They found that systolic blood pressure increased by $3.0 \mathrm{mmHg}$ and diastolic blood pressure increased by $1.5 \mathrm{mmHg}$, which may have been caused by the subject's increased blood pressure during sleep (systolic blood pressure, $+5.6 \mathrm{mmHg}$; diastolic blood pressure, $+1.9 \mathrm{mmHg}$ ), rather than the subject's increased blood pressure during wakefulness (systolic blood pressure, $+1.6 \mathrm{mmHg}$; diastolic blood pressure, $+1.4 \mathrm{mmHg}$ ). The above studies show that circadian rhythm disorder can increase the blood pressure levels of non-shift workers to varying degrees, and the increase in blood pressure is mainly due to the changes in blood pressure during sleep. In addition to the study on non-shift workers, Morris et al. (14) also studied chronic shift workers, and found that circadian rhythm disorder significantly increased 24-hour systolic and diastolic blood pressure by 1.4 and $0.8 \mathrm{mmHg}(\mathrm{P} \leq 0.038)$, respectively. The increase in 24-hour systolic blood pressure was considered to be due to increases in systolic blood pressure during the waking period $(+1.7 \mathrm{mmHg} ; \mathrm{P}=0.017)$, while the increase in 24-hour diastolic blood pressure was explained by the increase in diastolic blood pressure during sleep $(+1.8 \mathrm{mmHg} ; \mathrm{P}=0.005)$. Studies have shown that circadian rhythm disorder can lead to an increase in 24-hour blood pressure of chronic shift workers. However, the mechanism for the increase in systolic and diastolic blood pressure is different: the systolic blood pressure increase is caused during waking periods, while the diastolic blood pressure increase is caused during sleep. These results from previous studies show that for non-shift workers and chronic shift workers, circadian rhythm disorder leads to an increase of systolic and diastolic blood pressure, but with different mechanisms.

Increased blood pressure is a major risk factor for cardiovascular disease. Therefore, a small increase in blood pressure caused by circadian rhythm disorder may have important clinical significance (15). Circadian rhythm disorder itself can increase both systolic and diastolic blood pressure in non-shift and chronic shift workers. This provides relevant evidence that circadian rhythm disorder caused by shift work is a potential mechanism for inflammation and cardiovascular disease. Equally, it confirms that circadian rhythm disorder can cause adverse effects on cardiac metabolism due to acute events such as jet lag and chronic events such as changes in shift work.
In this study, research on the body fluid of subjects shows that circadian rhythm disorder causes a decrease in total body water and trunk water in women aged 31-40 years, and the decrease in total body water is particularly significant. Further analysis shows that the decrease in total body water is caused by the decrease of lower limb water and intracellular and extracellular fluid. Compared with the upper limb, the lower limb water levels were significantly lower. It is possible that this may be due to the lengthy sitting periods characteristic of the work environment of individuals with circadian rhythm disorder, which would affect the flow and metabolism of lower limb water. Results from the body muscle mass index of subjects indicate that circadian rhythm disorder causes a decrease in body muscle mass and trunk muscle mass in women aged $31-40$ years, and is especially significant in the reduction in body muscle mass. Further analysis reveals that the decrease in body muscle mass is attributed to the decrease in lower limb muscle mass. Compared with the changes in the muscle mass of the upper limbs, the muscle mass of the lower limbs was significantly reduced compared to the normal rhythm group, which may be due to the lengthy periods of sitting that characterize the work environment of individuals with circadian rhythm disorder. The above results show that the effect of circadian rhythm disorder on water and muscle mass changes is basically the same, indicating that circadian rhythm disorder affects water metabolism as well as muscle mass metabolism.

This study found that circadian rhythm disorder causes a significant increase in body fat in women aged 31-40 years, and mainly in visceral fat. The level of visceral fat and the percentage of body fat showed a tendency to be higher than the control group, while the amount of subcutaneous fat in the circadian rhythm disorder group was significantly decreased. The results indicated that circadian rhythm disorder causes an increase of body fat in women aged 31-40 years, especially of visceral fat, which is also related to their work characteristics. In this study, the circadian rhythm disorder group showed a significant increase in body fat and a decrease in weight for women in this age bracket. The results of Kushida et al. (16) have also shown that chronically sleep-deprived rats significantly increased their food intake, while simultaneously losing weight, which is confirmed by the results of this study. Further analysis attributed this finding to the significantly increased energy consumption of rats with chronic sleep deprivation, which led to the significant weight loss. Brock et al. (17) also found that long-term sleep deprivation can lead to an increase in 
energy consumption and weight loss. Many studies have shown that sleep deprivation changes energy expenditure, and that a lack of sleep will increase energy expenditure and reduce fat content. Except for corticosterone levels and body composition, most of the changes could be reversed by sleep recovery, and it has been reported that the weight loss caused by sleep deprivation is due to a decrease in fat (approximately $37 \%$ ), rather than a decrease in protein content (6). The reasons for the weight loss in the women in this study are complicated and may be due not only to the decrease in subcutaneous fat but also to changes in water, muscle and protein quality.

Previous studies have found that sleep disorders can also cause changes to metabolism. For example, Bonnet et al. (18) studied 12 normal young people for two 4-day periods and found that the metabolic rate of patients suffering from insomnia was significantly higher than that of healthy volunteers when measured by the maximum oxygen consumption. Everson et al. (19) found that rats with sleep deprivation showed an increase in the rate of fat consumption, which is mainly judged by the lack of fat in the rat's body during autopsy. Everson (19) and Brock (17) have shown that circadian rhythm disorder and sleep deprivation can lead to increased food intake and weight loss without causing nutritional waste or changes in intermediate metabolism. They hypothesized that the accelerated use of certain nutrients leads to the loss of body weight. Suchecki et al. (20) further confirm this view that circadian rhythm disorder and sleep deprivation can lead to increased food intake and weight loss, and the weight loss cannot be prevented even with a supplemental fat or sucrose diet. In addition, Papakonstantinou et al. (21) tested the effect of a high-fat diet consisting of fish oil as the main lipid source on sleep-deprived rats, and the results showed a decrease of fat tissue in rats, which was not changed by supplementing fat or fish oil. Bergmann et al. (22) studied the contribution of the hypothalamic-pituitary-adrenal axis (HPA), thyroid, and sympathetic adrenal system to the increase of energy expenditure by measuring plasma hormone levels, and reported that the increase in energy expenditure caused by circadian rhythm disorder may be mediated by the sympathetic nervous system.

In this study, we found that circadian rhythm disorder can lead to a decrease in the basal metabolic rate, protein quality and mineral quality in women aged $31-40$ years, and especially in significantly reduced protein quality. Kant et al. (23) conducted a 72-hour sleep-deprivation study on six young men and collected their urine for 5 consecutive 24-hour periods, and found that lack of sleep caused a significant increase in urea levels, and decreases in glucose and urine electrolytes, which is largely consistent with our findings. These previous studies have shown that sleep deprivation causes an increase in protein catabolism, indicating that lack of sleep can interfere with the body's normal metabolism.

Adrenal cortex hormones can increase blood pressure in humans and animals. However, the specific mechanism of the relationship between adrenal cortex hormones and altered renal function to other cardiovascular variables has not yet been fully elucidated. Physiological studies have found that cortisol and melatonin have opposite effects on blood pressure. Connell et al. (24) found that cortisol has a positive effect on the effect of adrenocorticotropic hormone (ACTH) to a large extent and increases blood pressure through corticotropin. Scheer et al. (12) found that regular use of melatonin reduced systolic and diastolic blood pressure by $6 \mathrm{mmHg}$ and $4 \mathrm{mmHg}$ during sleep, respectively. Melatonin had no effect on heart rate, but it caused increases of $15 \%$ and $25 \%$ in the circadian rhythm of systolic blood pressure and diastolic blood pressure, respectively. Other studies have found that in patients with essential hypertension, regular melatonin use before going to bed can significantly reduce the patient's nighttime blood pressure level. Cagnacci et al. (13) investigated whether long-term nighttime use of melatonin affected the rhythm of women's blood pressure. Their results indicated that melatonin use did not affect women's blood pressure during the day, nor did it cause significant changes in heart rate, but it significantly reduced women's nighttime systolic blood pressure $(-3.77 \pm 1.7 \mathrm{mmHg}, \mathrm{P}=0.0423)$, diastolic blood pressure $(-3.63 \pm 1.3 \mathrm{mmHg}, \mathrm{P}=0.0153)$, and mean blood pressure $(-3.71 \pm 1.3 \mathrm{mmHg}, \mathrm{P}=0.013)$. The decrease in blood pressure (systolic blood pressure) at night is almost coincident with the increase in melatonin, which may cause vasodilation and lower blood pressure. Prolonged use of melatonin may improve circadian blood pressure levels.

Morris et al. $(14,25)$ found that circadian rhythm disorder has no effect on the 24-hour average serum cortisol level, which indicates that the increases in blood pressure mediated by circadian rhythm disorder may not be related to changes in cortisol hormone levels. Therefore, we did not investigate cortisol hormone levels in this study, but we did measure melatonin levels. Our results showed that circadian rhythm disorder causes a significant drop in melatonin levels in women aged $31-40$ years, which is consistent with the study by Morris et al. (26), suggesting 
that exposure to light in an awake state will significantly reduce the production of melatonin. Melatonin can lower blood pressure, and circadian rhythm disorder can cause a decrease in the body's melatonin levels, so circadian rhythm disorder could increase blood pressure. The studies by Scheer (12) and Cagnacci et al. (13) also show that circadian rhythm disorder can increase blood pressure by causing a decrease in the body's melatonin level.

The influence of circadian rhythm disorder on early insulin secretion may be mediated by various mechanisms of the circadian system, among which the rhythm of endogenous physiological hormones can directly affect the compensation of human $\beta$-cells. In addition to the circadian adjustment of $\beta$-cell function, the circadian rhythm system may also affect glucose tolerance through insulin sensitivity and liver glucose output, which is similar to the pancreas. This study tested the insulin resistance of women with circadian rhythm disorder aged 31-40 years, and found a significant increase in their insulin resistance. This increase in insulin resistance indicates that the sensitivity of insulin is decreased, and the effect of insulin on glucose is weakened, leading to an increase in glucose concentration. Scheer et al. (10) simulated circadian rhythm disorder by scheduling a recurring 28 -hour day, and showed that despite an increase in insulin levels $(+22 \%, \mathrm{P}=0.006)$, circadian misalignment also induced a significant increase in glucose levels $(+6 \%, \mathrm{P}<0.001)$. It is worth noting that 3 out of 8 subjects in their study demonstrated a postprandial glucose response, which is typical of a prediabetic state. Studies have shown that living in dim light with a 28 -hour behavioral cycle can lead to an increase of glucose concentrations after meals. Morris et al. (26) found that circadian disruption can cause a $6 \%$ increase in postprandial glucose, thereby reducing glucose tolerance. These changes are caused by different mechanisms. On the one hand, it can be caused by inhibition of the pancreatic $\beta$-cell function at night when the rhythm is normal. On the other hand, it may be achieved by the reduction of insulin sensitivity due to circadian rhythm disorder. Studies have also shown that the effects of circadian rhythm disorder on glucose tolerance are mainly regulated by decreased insulin sensitivity, rather than decreased pancreatic $\beta$-cell function. These results suggest a mechanism for the increased risk of diabetes among shift workers. Decreased insulin sensitivity is a key defect in the early development of type 2 diabetes. Therefore, the increase in insulin resistance in women with circadian rhythm disorder in this study is consistent with results from previous studies where circadian misalignment leads to decreased insulin sensitivity. Gan et al. (27) showed that shift work is associated with an increased risk of diabetes, especially in male shift workers. Since circadian rhythm disorder is associated with the occurrence of type 2 diabetes, Coomans et al. (28) studied the suprachiasmatic nucleus (the central pacemaker of the circadian system), which plays a direct role in the development of insulin resistance. Their results demonstrated that the dysfunction of the suprachiasmatic nucleus plays an important role in the imbalance of energy, and that in genetically intact animals, an absence of central clock activity may lead to the development of insulin resistance. Shi et al. (29) also provided evidence for the circadian rhythm of insulin action, by demonstrating that disturbance of the natural rhythm of insulin action destroys the rhythm of insulin-sensitive tissues, thereby making animals prone to insulin resistance and obesity. The above results suggest a possible mechanism for humans with circadian rhythm disorder to be more prone to impaired glucose metabolism and type 2 diabetes.

In short, in this study we demonstrated that circadian rhythm disorder causes an increase in women's blood pressure, and a decrease of body water, especially lower limb water and intracellular fluids. Circadian rhythm disorder also results in decreased muscle mass and protein quality, as well as increased visceral fat levels in women. Additionally, it also causes a decrease in melatonin and an increase in insulin resistance. There are both similarities and differences in the current findings compared with previous research results, which may be explained by the following two reasons: firstly, studies have used different experimental subjects, with some research based on animal subjects, and others based on human subjects. Secondly, differences in the experimental design of studies investigating circadian rhythm misalignment and disorder may have led to differences in research outcomes.

In conclusion, the present study has shown that circadian rhythm disorder can cause changes in body composition, melatonin, and insulin resistance in women aged 31-40 years, and therefore disruption to the circadian rhythm might be a factor for increasing the risk of cardiovascular disease and diabetes in women in this age bracket.

\section{Acknowledgments}

Funding: This study was approved by the Foundation for the Introduction and Education program for Young Talent in Shandong Universities (72), and the China Postdoctoral Science Foundation (2014M560538). 


\section{Footnote}

Reporting Checklist: The authors have completed the MDAR checklist. Available at http://dx.doi.org/10.21037/apm-202267

Data Sharing Statement: Available at http://dx.doi. org/10.21037/apm-20-2267

Conflicts of Interest: All authors have completed the ICMJE uniform disclosure form (available at http://dx.doi. org/10.21037/apm-20-2267). The authors have no conflicts of interest to declare.

Ethical Statement: The authors are accountable for all aspects of the work in ensuring that questions related to the accuracy or integrity of any part of the work are appropriately investigated and resolved. All participants were required to complete a sleep status questionnaire and provided informed consent. The experiment was approved by the school ethics committee of Jiangsu Normal University. All procedures performed in this study involving human participants were in accordance with the Declaration of Helsinki (as revised in 2013).

Open Access Statement: This is an Open Access article distributed in accordance with the Creative Commons Attribution-NonCommercial-NoDerivs 4.0 International License (CC BY-NC-ND 4.0), which permits the noncommercial replication and distribution of the article with the strict proviso that no changes or edits are made and the original work is properly cited (including links to both the formal publication through the relevant DOI and the license). See: https://creativecommons.org/licenses/by-nc-nd/4.0/.

\section{References}

1. Lima AM, de Bruin VM, Rios ER, et al. Differential effects of paradoxical sleep deprivation on memory and oxidative stress. Naunyn Schmiedebergs Arch Pharmacol 2014;387:399-406.

2. Grandner MA, Chakravorty S, Perlis ML, et al. Habitual sleep duration associated with self-reported and objectively determined cardiometabolic risk factors. Sleep Med 2014;15:42-50.

3. Hui M, Li Y, Ye J, et al. Obstructive sleep apnea-hypopnea syndrome (OSAHS) comorbid with diabetes rather than OSAHS alone serves an independent risk factor for chronic kidney disease (CKD). Ann Palliat Med 2020;9:858-69.

4. Martins PJ, Marques MS, Tufik S, et al. Orexin activation precedes increased NPY expression, hyperphagia, and metabolic changes in response to sleep deprivation. Am J Physiol Endocrinol Metab 2010;298:E726-34.

5. Galvão MO, Sinigaglia-Coimbra R, Kawakami SE, et al. Paradoxical sleep deprivation activates hypothalamic nuclei that regulate food intake and stress response. Psychoneuroendocrinology 2009;34:1176-83.

6. Hipólide DC, Suchecki D, Pimentel de Carvalho Pinto A, et al. Paradoxical sleep deprivation and sleep recovery: effects on the hypothalamic-pituitary-adrenal axis activity, energy balance and body composition of rats. J Neuroendocrinol 2006;18:231-8.

7. Koban M, Stewart CV. Effects of age on recovery of body weight following REM sleep deprivation of rats. Physiol Behav 2006;87:1-6.

8. Štefan L, Sporiš G, Krističević T, et al. The associations between sleep duration and sleep quality with self-rated health in young adults: a population-based study. Int J Adolesc Med Health 2018. [Epub ahead of print]

9. Taheri S, Lin L, Austin D, et al. Short sleep duration is associated with reduced leptin, elevated ghrelin, and increased body mass index. PLoS Med 2004;1:e62.

10. Scheer FA, Hilton MF, Mantzoros CS, et al. Adverse metabolic and cardiovascular consequences of circadian misalignment. Proc Natl Acad Sci USA 2009;106:4453-8.

11. Morris CJ, Purvis TE, Hu K, et al. Circadian misalignment increases cardiovascular disease risk factors in humans. Proc Natl Acad Sci USA 2016;113:E1402-11.

12. Scheer FA, Van Montfrans GA, van Someren EJ, et al. Daily nighttime melatonin reduces blood pressure in male patients with essential hypertension. Hypertension 2004;43:192-7.

13. Cagnacci A, Cannoletta M, Renzi A, et al. Prolonged melatonin administration decreases nocturnal blood pressure in women. Am J Hypertens 2005;18:1614-8.

14. Morris CJ, Purvis TE, Mistretta J, et al. Circadian Misalignment Increases C-Reactive Protein and Blood Pressure in Chronic Shift Workers. J Biol Rhythms 2017;32:154-64.

15. Stamler J, Stamler R, Neaton JD, et al. Blood pressure, systolic and diastolic, and cardiovascular risks. US population data. Arch Intern Med 1993;153:598-615.

16. Kushida CA, Bergmann BM, Rechtschaffen A, et al. Sleep deprivation in the rat: IV. Paradoxical sleep deprivation. Sleep 1989;12:22-30.

17. Brock JW, Farooqui SM, Ross KD, et al. Stress-related 
behavior and central norepinephrine concentrations in the REM sleep-deprived rat. Physiol Behav 1994;55:997-1003.

18. Bonnet MH, Arand DL. Metabolic rate and the restorative function of sleep. Physiol Behav 1996;59:777-82.

19. Everson CA, Bergmann BM, Rechtschaffen A, et al. Sleep deprivation in the rat: III. Total sleep deprivation. Sleep 1989;12:13-21.

20. Suchecki D, Antunes J, Tufik S, et al. Palatable solutions during paradoxical sleep deprivation: reduction of hypothalamic-pituitary-adrenal axis activity and lack of effect on energy imbalance. J Neuroendocrinol 2003;15:815-21.

21. Papakonstantinou E, Ryan DH, Harris RB, et al. Dietary fish oil does not protect rats exposed to restraint or sleep deprivation stress. Physiol Behav 2003;78:759-65.

22. Bergmann BM, Everson CA, Kushida CA, et al. Sleep deprivation in the rat: V. Energy use and mediation. Sleep 1989;12:31-41.

23. Kant GJ, Genser SG, Thorne DR, et al. Effects of 72 hour sleep deprivation on urinary cortisol and indices of metabolism. Sleep 1984;7:142-6.

24. Connell JM, Whitworth JA, Davies DL, et al. Effects of ACTH and cortisol administration on blood pressure,

Cite this article as: Meng R, Cao Y, Kong Y, Wang K, Yang Z, Jia Y, Dong C, Duan H, Han M. Effects of circadian rhythm disorder on body composition in women aged 31-40 years. Ann Palliat Med 2021;10(1):340-349. doi: 10.21037/apm-20-2267 electrolyte metabolism, atrial natriuretic peptide and renal function in normal man. J Hypertens 1987;5:425-33

25. Morris CJ, Purvis TE, Mistretta J, et al. Effects of the Internal Circadian System and Circadian Misalignment on Glucose Tolerance in Chronic Shift Workers. J Clin Endocrinol Metab 2016;101:1066-74.

26. Morris CJ, Yang JN, Garcia JI, et al. Endogenous circadian system and circadian misalignment impact glucose tolerance via separate mechanisms in humans. Proc Natl Acad Sci USA 2015;112:E2225-34.

27. Gan Y, Yang C, Tong X, et al. Shift work and diabetes mellitus: a meta-analysis of observational studies. Occup Environ Med 2015;72:72-8.

28. Coomans CP, van den Berg SA, Lucassen EA, et al. The suprachiasmatic nucleus controls circadian energy metabolism and hepatic insulin sensitivity. Diabetes 2013;62:1102-8.

29. Shi SQ, Ansari TS, McGuinness OP, et al. Circadian disruption leads to insulin resistance and obesity. Curr Biol 2013;23:372-81.

(English Language Editor: D. Fitzgerald) 